\title{
Constraints of Behavioural Inheritance
}

\author{
E. E. Roubtsova, S. A. Roubtsov` \\ Technical University Eindhoven, Den Dolech 2, P.O. Box 513, \\ 5600MB Eindhoven, The Netherlands. \\ E.Roubtsova@tue.nl, S.Roubtsov@tue.nl
}

\begin{abstract}
We present an approach to component inheritance and reuse which closes the gap between architectural design and process-oriented approaches. To apply inheritance checks in design and verification of a system, one should consider an inheritance relation as a property of the system and specify it as an inheritance constraint. To specify the inheritance constraints we offer a logic of behavioural inheritance. In a UML profile with the process tree semantics we show how to use this logic for architectural design and for verification with respect to the specified inheritance constraint.

Keywords: Constraint of behavioural inheritance, logic of behavioural inheritance, process tree semantics, UML profile, behaviour specification reuse.
\end{abstract}

\section{Introduction}

Inheritance of components is one of the accepted instruments for reuse of components in architectural design $[1,2]$. However, in architectural approaches, like CATALYSIS [2] or ISpec [3]), and in Architecture Description Languages (ADLs), like Rapide, C2 [1] or Koala [4], the notion of component inheritance is a predefined part of the underlying metamodel. The support of the system evolution in those approaches is restricted by structural subtyping [1] of components. However, the structural subtyping relation allows defining an infinite set of behaviour inheritance relations on parent and child components. The behaviour of a parent can be repeated in a child before or after some new behaviour fragments, it can be repeated for a specific part of the child behaviour or it can be divided into parts by some new behaviour fragments. Thus, a component-inheritor specification can satisfy one of the behavioural inheritance relations and not satisfy another. In practice, this usually becomes clear only after producing and testing the behaviour specification of a component-inheritor, because the current approaches to architectural design do not direct and help designers to think about the necessary behavioural inheritance relation in advance. Consequently, this causes semantic mistakes in architectural design.

There are process-oriented architectural approaches, like SADL [1], which represent ordering constraints among sub-processes of a process. Those approaches come closer to the problem of component behavioural inheritance. But

\footnotetext{
* The research of S.A.Roubtsov was partly supported by PROGRESS (STW EES5141) and EMPRESS (ITEA 01003) projects.
} 
the component behavioural inheritance [5] is defined for the process approaches as a finite set of potential inheritance relations on processes representing components. The relations are classified on the basis of the back transformation of a component-inheritor specification to a component-parent specification. So, if it is possible to transform a component-inheritor specification to a componentparent specification, then a designer can prove that the inheritance of some type is correct. However, the process-oriented approaches do not give us any clue of where to use one or another type of behavioural inheritance relations and how to specify such relations, i.e. the notion of behavioural inheritance given in the process theory [5] has little connection with the tasks of architectural design.

In this paper we present an approach to component inheritance and reuse which closes the gap between architectural design and process-oriented approaches. We suggest that, to apply inheritance checks in design and verification of a system, one should consider an inheritance relation as a property of the system. Moreover, we define such a property in terms of architectural design. Any particular type of behavioural inheritance cannot be correct or incorrect in itself. It is for a designer to decide which type of possible behavioural inheritance relations fits the case in question and, then, to prove that such a type holds in the design specification.

In verification methods, specification of properties is always based on an abstraction chosen for the system specification $[6,7]$. Our system is a component exchanging messages with the environment and other components. We consider a behavioural pattern containing sequences, alternatives and cycles of such messages (e.g., operation calls and returns) as a unit of system specification.

When a new component inherits a parent component, we should give a specification of how exactly the parent's behavioural pattern should be reused. Designers may have different ideas on how to reuse a particular behavioural pattern. One case demands establishing some conditions on reuse of the pattern, another case - fulfilling the pattern for all alternatives. So, there is no sole behavioural inheritance specification, but an infinite set of them. The chosen inheritance specification should become a property of the inheritor's design specification and this property must be kept.

Consequently, we consider behaviour inheritance relations as constraints. The standard constraint language OCL [8] is not suitable for the specification of behavioural inheritance relations because it does not manipulate processes as abstract elements. Because of that we offer a logic of behavioural inheritance to define inheritance constraints. Constraint languages can be extended on the basis of this logic.

An inheritance constraint describes how the process of a component-parent can appear in the process of a component-child. We consider the componentchild to be a correct inheritor of the component-parent with respect to the specified behavioural inheritance constraint if the inheritance constraint holds for the process of the component-child. A predicate of the logic of behavioural inheritance represents the place of the parents's process tree in the child's process tree. A process tree is an abstract variant of a computation tree. Formulas of our 
logic describe properties of this computation tree. So, our logic is a computation tree logic with a process interpretation.

The logic of behavioural inheritance allows designers to specify what kind of behavioural inheritance they would like to achieve. Moreover, the logic defines types of constraints as logical units and allows us to put a corresponding technique to each type of constraint to prove that this constraint holds. So, the logic provides methodological support for reuse of component behaviour in architectural design.

The paper is organized as follows. In Section 2 we define a component behavioural pattern as a process and a corresponding process tree. An example of the process tree semantics is given for a component specification profile in the UML. In this profile we also demonstrate specification of components using inheritance. Section 3 describes our logic of behavioural inheritance and explains how to use this logic for architectural design and for proving correctness of component behavioural inheritance. Section 4 concludes the paper.

\section{A Behavioural Pattern as a Process Tree}

In our approach, a component specification is a process $p$ of type

$$
P=(A, S P, T)[5], p \in P, \text { where }
$$

- $A$ is a finite set of actions.

- $S P=\left\{s p, s p_{1}, s p_{2}, \ldots, s p_{F}\right\}$ is a finite set of abstract states from the unique initial state $s p$ to the unique final state $s p_{F}$.

- $T$ is a set of transitions. Transition $t \in T$ defines a triple $\left(s p^{\prime}, s p^{\prime \prime}, a\right)$, such that state $s p^{\prime \prime}$ is reachable from state $s p^{\prime}$ as a result of action $a \in A: s p^{\prime} \stackrel{a}{\Longrightarrow} s p^{\prime \prime}$.

We construct a process tree for a component behaviour specification.

A process tree is a process graph [9] $G_{p}=(N, E)$ which has a unique path from the node root to every other node. Each process tree corresponds to its process $p$ so that:

- Each node $n \in N$ of the process tree corresponds to an abstract state from set $S P$. The root corresponds to the initial state $s p$.

- Each node, except final nodes, is labelled by the process name which represents the process starting from the state corresponding to this node. Final nodes, labelled by $\sqrt{ }$, correspond to the final state $s p_{F}$.

- Each edge $e=\left(n^{\prime}, n^{\prime \prime}, a\right) \in E$ of the process tree corresponds to a transition from set $T$. An edge is labelled by an action $a \in A$. Edges to final nodes carry the termination label $\downarrow$.

Thus, a path in a process tree is a sequence of arcs

$$
\left(\left(n_{1}, n_{2}, a_{1}\right),\left(n_{2}, n_{3}, a_{2}\right), \ldots,\left(n_{m-1}, \sqrt{ }, \downarrow\right)\right) .
$$

There is a unique sequence of actions that corresponds to each path: $a_{1}, a_{2}, \ldots, a_{m-1} \downarrow$. A path which starts from the root is called a root path. The 
node labels, the final nodes labelled by $\sqrt{ }$ and the edges labelled by $\downarrow$ can be omitted [9] to simplify process tree graphical representation.

If a component behaviour specification contains cycles, then we represent each cycle by two paths: one path for the cycle's body and the other path for the cycle's exit. Repeated cycle's bodies are replaced by dots: "...".

Computation trees similar to our process tree are widely accepted as internal models for specification languages. Computation tree semantics has been defined for automata specifications $[6,10]$ and for UML profiles containing statecharts [7]. In the next subsection, we define a process tree semantics for our UML profile for component specification and reuse.

\subsection{Process Tree Semantics for Our UML Profile}

Our UML profile is one in the family of UML-like languages [11]. We specify a process of a component in a role language. This role language is represented in an identified subset of the UML metamodel [12].

The elements of a process are specified in terms of roles communicating via interfaces. A role is a UML class with stereotype $\ll$ Role $\gg$. In general, a role can have several players (instances), but we do not refer to players in this paper. An interface comprises a semantically close group of operations of a role. An interface is always provided by a particular role which implements operations of this interface. The interface can be required by other roles or, maybe, by the role itself. These provide and require relations specify actions of our process graph. To refer to a particular action we use the conventional "dot"-notation. In this notation an operation call, for example, is denoted as

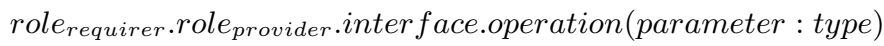

and its return (callback) as

role $_{\text {requirer.role }}$ provider.inter face.operation(parameter : type) : result.

The notation above provides uniqueness of operation names within the entire component specification. In all examples of this paper each interface has exactly one operation with different results. So, it is possible to use the shortened notation for action names:

role $_{\text {requirer }}$.role provider. $_{\text {interf face }}($ parameter : type)

role $_{\text {requirer.role }}$ provider.interface (parameter : type) : result.

The actions and the component behavioural pattern built from such actions are specified by an interface-role diagram $I R$ and a set of sequence diagrams $S_{1} \ldots S_{k}$.

An interface-role diagram (Fig. 1a, 2) is a graph $I R=(R, I, P I, R I, R R)$ with two kinds of nodes and three kinds of relations:

- $R$ is a finite set of roles. Each role $r \in R$ is depicted by a box.

- $I$ is a finite set of interfaces depicted by circles. In this paper, each interface $i \in I$ has one operation identified by the interface name. Each operation has a set of results $\operatorname{Res}_{i}$. 
- PI $\subseteq\{(r, i) \mid r \in R, i \in I\}$ is a provide relation on roles and interfaces. Each role provides a finite set of interfaces. An element of the relation is depicted by a solid line between a role and an interface.

- $R I \subseteq\left\{\left(r^{\prime},(r, i)\right) \mid r^{\prime}, r \in R, i \in I,(r, i) \in P I\right\}$ is a require relation on roles and interfaces. Each role requires a finite set of provided interfaces. An element of the relation is drawn by a dash arrow connecting a role and a provided interface. The arrow is directed to the interface.

- $R R \subseteq\left\{\left(r, r^{\prime}\right) \mid r, r^{\prime} \in R\right\}$ is a relation of inheritance on the set of roles. An element of the relation is shown by a solid line with the triangle end $r^{\prime} \rightarrow r$ directed from role-child $r^{\prime}$ to role-parent $r$.

A sequence diagram (Fig. 1b) is a UML sequence diagram [8]

$$
S=\left(B, A_{s}, \aleph \rightarrow A_{s}\right)
$$

- $B=\left\{b_{i}\right\}$ is a set of boxes with dash lines drawn down from each box and representing the time dimension. In our profile, box $b_{i} \in B$ represents a player (an instance) of a role from the interface-role diagram. We have assumed that each role has only one player, so a box represents a role.

- $A_{s}$, is a set of labelled arcs.

An $\operatorname{arc}\left(b_{i}, b_{j}, l\right) \in A_{s}$ is depicted as an arrow that connects the dash line running from box $b_{i}$ to the dash line running from box $b_{j}$. An arc has a label $l$ which represents an operation, for example,

$l=$ interface.operation (parameter) for an operation call or

$l=$ interface.operation (parameter) $:$ result for an operation return

(or $l=$ interface(parameter) and

$l=$ interface (parameter $):$ result , if each interface has only one operation.)

- $\aleph \rightarrow A_{s}, \aleph=\{1,2,3 \ldots\}$ is a function defined on a subset of natural numbers that orders arcs.

Process tree. From each specification in the described above profile we construct a process tree.

$S$-tree. A sequence diagram corresponds to a process tree $G=(N, E)$ which contains one path. We name such a tree $s$-tree: $e_{1}, \ldots, e_{k}=\left(n_{1}, n_{2}, a_{1}\right), \ldots,\left(n_{k}, n_{k+1}, a_{k}\right)$, where $e_{x}=\left(n_{x}, n_{x+1}, a_{x}\right), x=1, \ldots, k, a_{x}=x \rightarrow\left(b_{i}, b_{j}, l\right),\left(b_{i}, b_{j}, l\right) \in A_{s}$.

Operation Fusion: Let a process tree and an s-tree be given.

- If a root path of the process tree and a root subsequence of the s-tree have the same sequence of labels of arcs, then this path and this subsequence are fused, i.e. joined in one path.

- The first arc of the s-tree, the label of which differs from the label of the current arc in the root path of the process tree, starts a new branch from the last fused node of the process tree.

Process tree construction.

1. S-tree constructed from a sequence diagram is a process tree.

2. The result of the fusion of a process tree with an s-tree is a process tree.

3. There are no other process trees.

The detailed description of the algorithms can be found in [13]. 
For a component specification in our profile, the set $E$ of arcs of the process tree $G_{p}=(N, E)$ is exactly defined from the set of arcs of all the sequence diagrams: $E=A_{s_{1}} \cup \cdots \cup A_{s_{k}}$. In turn, the set of arcs of all the sequence diagrams $A_{s_{1}} \cup \cdots \cup A_{s_{k}}$ is a multiset on the require relation set $R I$ from the interface-role diagram of this component (some operations can be called several times). The process tree of a component can be easily transformed back to its sequence diagrams: each root path of the process tree is mapped onto an s-tree corresponding to a sequence diagram. In the next subsection, we give an example of a component specification in our profile.

\subsection{Specification of Component Web Service in Our UML Profile}

Let us consider an abstract component Web Service. Like most services on the Web, this service sends back some data in response to a user's request. (Even if you buy something, Internet itself never sends you goods, it only promises you to send goods later.) The component provides an opportunity to choose one of Web services from a list. Usually, before responding, the server asks the client for some additional information. For example, to get access to search engines the client should identify a kind of information to be retrieved; to buy things in an e-shop the customer should choose them and provide data that guarantees the purchase, and so on. In all cases the process is essentially the same; the differences (what kind of response is required, what additional information is needed and how to ask it) can be hidden in the server's software. This allows us to consider such an adjustable service as a reusable component in the Web service interaction model. Fig. 1 shows the specification of component Web Service, which we intend to reuse.

The interface-role diagram of component Web Service is shown in Fig. 1a. Role Web Server provides two interfaces: IServiceList : $\{I D$ : integer $\}$, which returns identifier $I D$ of a chosen service and IService(ID:integer) : $\{$ true, false $\}$, which has two return values: true that means the successful result of a service request and false that means the unsuccessful result.

Role Web Client requires interfaces of Web Server and provides interface IFillForm(Form:structure) : \{correct structure, incorrect structure $\}$. Two types of the return value indicate two possible results of interaction via this interface: the correct structure, if the form is filled in correctly, and the incorrect structure, if some fields of the form are filled in incorrectly.

Two sequence diagrams present the behavioural model of component Web Service (Fig. 1b). The first sequence diagram models the successful behavioural pattern. Web Client chooses the service defined by parameter $I D$ from the list and requests this service. In response Web Server sends back the form defined by the parameter Form to be filled in. After the correct data structure has been filled in and sent to Web Server, it fulfils the service (return value true) and the session ends. The second sequence diagram corresponds to the case when the client's data for some reason is inappropriate. In such a case Web Server responds by the false return value and requests the data again. 


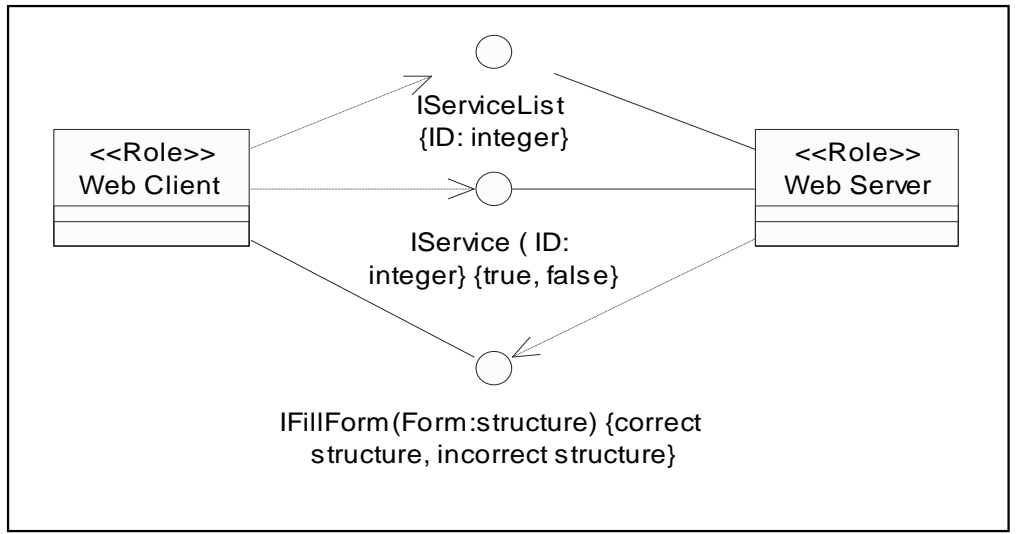

a)

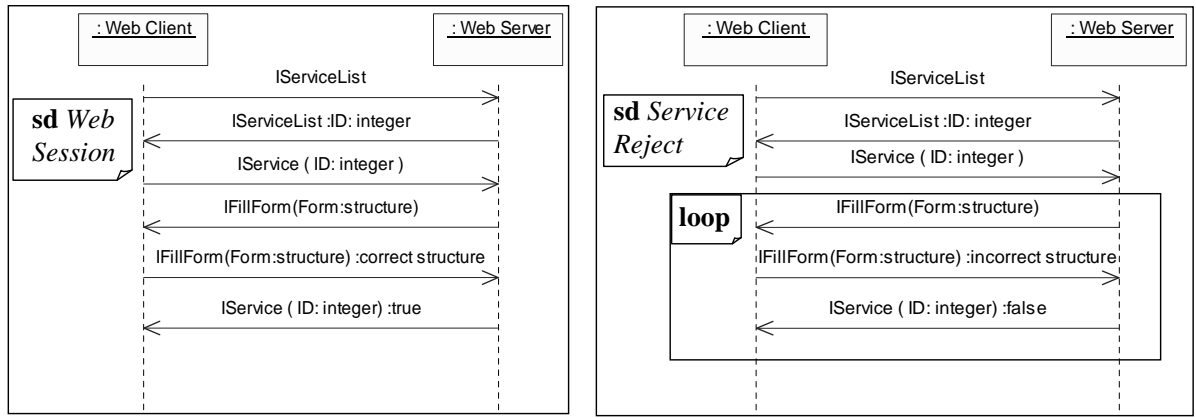

b)

$\|$

a1

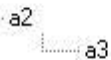

List of actions:

a1 - WebClient.WebServer.IServiceList

a2 - WebClient.WebServer.IServiceList:ID:integer

a3 - WebClient.WebServer. IService(ID:integer)

a4 - WebServer.WebClient. IFillForm(Form:structure)

a5 - WebServer.WebClient.IFillForm(Form:structure):correctstructure

a6 - WebClient.WebServer.IService(ID:integer $\}$ :true

a7 - WebServer.WebClient.IFillForm(Form:structure):incorrectstructure

a8 - WebClient.WebServer.IService(ID:integer $\}$ :false

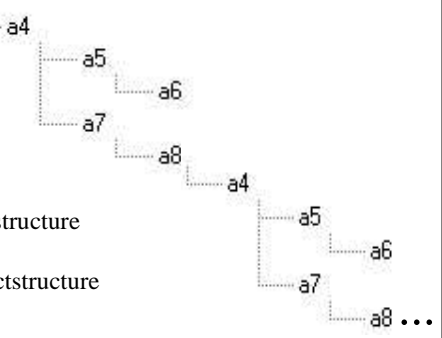

c)

Fig. 1. The specification of component Web Service: a) interface-role diagram; b) sequence diagrams; c) process tree 
This is a usual behavioural pattern for Web services: you can escape repetition of data requests by cancelling the connection or navigating to another Web page. Of course, more robust variants exist, e.g., a client may also be allowed to cancel the session within a requested form, the number of attempts may be restricted, and so on. However, in our component we have decided to rely on the common Web ideology. So, here we have a cycle, which is depicted by operator loop from the UML2.0 notation [14] newly adapted by OMG group.

The process tree representing behaviour of component Web Service is shown in Fig. 1c. In this figure and later we show action names only; the node labels, the final nodes labelled by $\sqrt{ }$ and the edges labelled by $\downarrow$ are omitted to simplify the picture.

From action $a_{4}=$ WebServer.WebClient.IFillForm(Form : structure) (the request from Web Server to Web Client to fill in the Form) the process tree branches out: one branch ends after the service has been completed and the other runs a possibly infinite cycle. Let the name of the process of component Web Service be $p=W e b$ Service. We shall use this process as a unit of behaviour to inherit from.

\subsection{Component Specification by Inheritance}

Inheritance of components in interface-role diagrams is specified by the inheritance relation on roles $R R$.

Definition 1. Let two interface-role diagrams be given:

$$
I R_{p}=\left(R_{p}, I_{p}, P I_{p}, R I_{p}, R R_{p}\right), I R_{q}=\left(R_{q}, I_{q}, P I_{q}, R I_{q}, R R_{q}\right) .
$$

Interface-role diagram $I R_{q}$ inherits interface-role diagram $I R_{p}$, if and only if there is an interface-role diagram $I R_{\text {new }}=\left(R_{\text {new }}, I_{\text {new }}, P I_{\text {new }}, R I_{\text {new }}, R R_{\text {new }}\right)$, (Fig. 2) such that

1. $R_{q}=R_{p} \cup R_{\text {new }}$. Role sets $R_{p}$ and $R_{\text {new }}$ are disjoint, i.e. $R_{p} \cap R_{\text {new }}=\emptyset$.

2. $I_{q}=I_{p} \cup I_{\text {new }}$. Interface sets $I_{p}, I_{\text {new }}$ are disjoint, i.e. $I_{p} \cap I_{\text {new }}=\emptyset$.

3. Only new roles can inherit roles of the parent interface-role diagram. Parent roles cannot inherit new roles.

$R R_{q}=R R_{p} \cup R R_{d}$, where

$R R_{d}=\left\{\left(r_{p}, r_{d}\right) \mid r_{p} \in R_{p} \wedge r_{d} \in R_{d} \wedge R_{d} \subseteq R_{n e w}, \wedge r_{d}-\triangleright r_{p}\right\}, \quad R R_{d} \neq \emptyset$.

So, the relation $R R_{d}$ defines subset of roles $R_{d} \subseteq R_{\text {new }}$ which have parents in the set $R_{p}$.

4. Elements of the provide relation from roles-parents are duplicated in the interfacerole diagram $I R_{q}$ by roles-inheritors because of the inheritance relation $R R_{d}$.

$P I_{q}=P I_{p} \cup P I_{d} \cup P I_{\text {new }}$,

$\left.P I_{d}=\left\{\left(r_{d}, i\right) \mid \quad r_{d} \in R_{d} \wedge \quad i \in I_{p} \wedge\left(\exists r \in R_{p} \mid r_{d} \rightarrow \triangleright r \wedge(r, i) \in P I_{p}\right)\right)\right\}$.

Sets $P I_{p}$ and $\left(P I_{d} \cup P I_{\text {new }}\right)$ are disjoint, i.e. $P I_{p} \cap\left(P I_{d} \cup P I_{\text {new }}\right)=\emptyset$.

5. Elements $(x,(r, i))$ of the require relation $R I_{p}$ are duplicated in the interface-role diagram $I R_{q}$ if both role $r$ that provides interface $i$ and role $x$ that requires interface $i$ are inherited.

$R I_{q}=R I_{p} \cup R I_{\text {new }} \cup R I_{d}$, where

$R I_{d}=\left\{\left(x_{d},\left(r_{d}, i\right)\right) \mid r_{d}, x_{d} \in R_{d} \wedge i \in I_{p} \wedge\left(\exists r, x \in R_{p}, \mid\left(r_{d}-\triangleright r \wedge x_{d}-\triangleright x \wedge\right.\right.\right.$

$\left.\left.\left.(r, i) \in P I_{p} \wedge(x,(r, i)) \in R I_{p}\right)\right)\right\}$.

Sets $R I_{p}$ and $\left(R I_{\text {new }} \cup R I_{d}\right)$ are disjoint, i.e. $R I_{p} \cap\left(R I_{\text {new }} \cup R I_{d}\right)=\emptyset$. 
The inheritance relation $R R_{d}$ in the interface-role diagram $I R_{q}$ defines a duplicating function $\rho_{R I_{p}}^{R I_{d}}$ which maps the parent require relations onto the subset of the child require relations. Elements of the set $R I_{d}$ are not depicted in the interface-role diagram $I R_{n e w}$, although they are inherited from the parent.

As we have mentioned already, a name of an action in our specification includes names of the role-provider and the role-requirer. If both the role provider

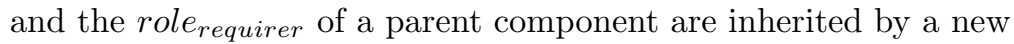

role inheritor-of-the-provider $_{\text {and a new role }}$ inheritor-of-the-requirer correspondingly, then the actions defined by the role-provider and the role-requirer are inherited by the new component.

The sequence diagrams of the child component are constructed from the actions specified by its interface-role diagram. If the parent process is inherited, then its actions are renamed using the duplicating function $\rho_{R I_{p}}^{R I_{d}}$ and the parent process $p$ is transformed to the duplicated process $p^{\prime}=\rho_{R I_{p}}^{R I_{d}}(p)$ which is equivalent to $p$ under duplicating. Here and later we indicate a duplicated process by the prime mark (e.g. $\left.p^{\prime}\right)$.

\subsection{An Example of Component Specification by Inheritance}

Let us design component Corporative Provider which inherits component Web Service. The new component enables all the possibilities of component Web Service but "for members only". Membership is supposed to be obtained somewhere outside the Web, using security ID cards, for example. For non-members a corporate server should simply deny access. Of course, the alternative behaviour is quite rudimentary but it could easily be extended by some predefined service, a kind of survey for guests, for example. The behaviour of component Web Service should be inherited by the new component just in one case, for a corporate member. Therefore, the predefined process of a membership check must come before the choice of a service.

Fig. 2 shows the interface-role diagram of component Corporative Provider. Two new roles Member and Corporative Server interact via the two new interfaces IMemberAccess and IMemberInfo: Member asks for access and afterward Corporative Server requests information via IMemberInfo. Depending on the return value of IMemberInfo role Corporative Server allows or denies access.

Role Member inherits role Web Client; role Corporative Server inherits Web Server from component Web Service. This means that roles Member and Corporative Server are able to perform all actions which roles Web Client and Web Server use in communication. So, the new component Corporative Provider is able to utilize the complete parent behaviour of component Web Service. This is depicted in sequence diagrams shown in Fig. 3. All three sequence diagrams are started by obligatory process $q=$ Check Membership. The first two sequence diagrams are started with subprocess $s=$ CorrectMembership after which the inherited process $p^{\prime}=W$ ebService ${ }^{\prime}=\rho_{R I_{p}}^{R I_{d}}(p)$ (which is equivalent to the parent process $p=W e b$ Service under duplication) fulfils itself. The third sequence 


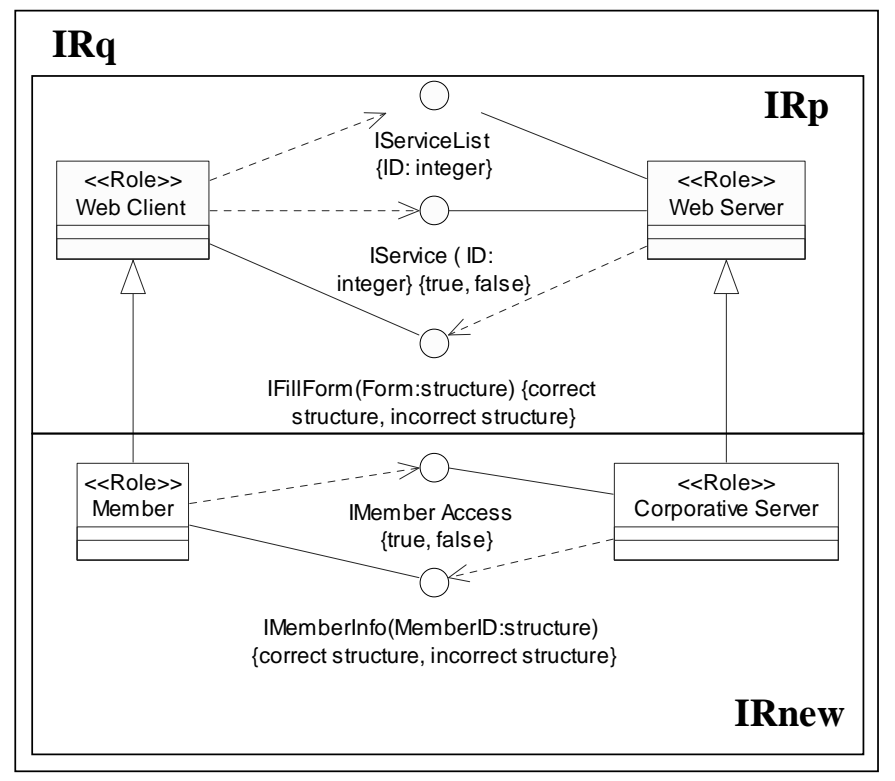

Fig. 2. The interface-role diagram of component Corporative Provider

diagram in Fig. 3 shows the alternative process Incorrect Memberships, i.e. the membership is not confirmed.

Thus, our UML profile allows a designer to specify the processes that should be inherited. The next section discusses how to help him/her formally specify the goal of inheritance and how to prove that this goal is achieved.

\section{A Logic of Behavioural Inheritance}

\subsection{An Existential Definition of Behavioural Inheritance}

Behavioural inheritance has been defined in [5]. Generalizing this definition we have the following:

Process $c$ inherits process $p$ if and only if there exist disjoint sets of actions $H, I \subseteq A_{c} \backslash A_{p}$, such that it is possible to derive process $p$ from process $c$ by

- blocking actions from $H$ in process $c$ using blocking function $\delta_{H}(c)$;

$\delta_{H}(c): P \rightarrow P$

$a \in H \rightarrow \delta_{H}(a)=\delta ; \delta$ is a blocked action;

$a \notin H \rightarrow \delta_{H}(a)=a$;

- hiding actions from $I$ in process $\delta_{H}(c)$ using hiding function $\tau_{I}\left(\delta_{H}(c)\right)$;

$\tau_{I}\left(\delta_{H}(c)\right): P \rightarrow P$

$a \in I \rightarrow \tau_{H}(a)=\tau ; \tau$ is a hidden action;

$a \notin I \rightarrow \tau_{I}(a)=a$ 

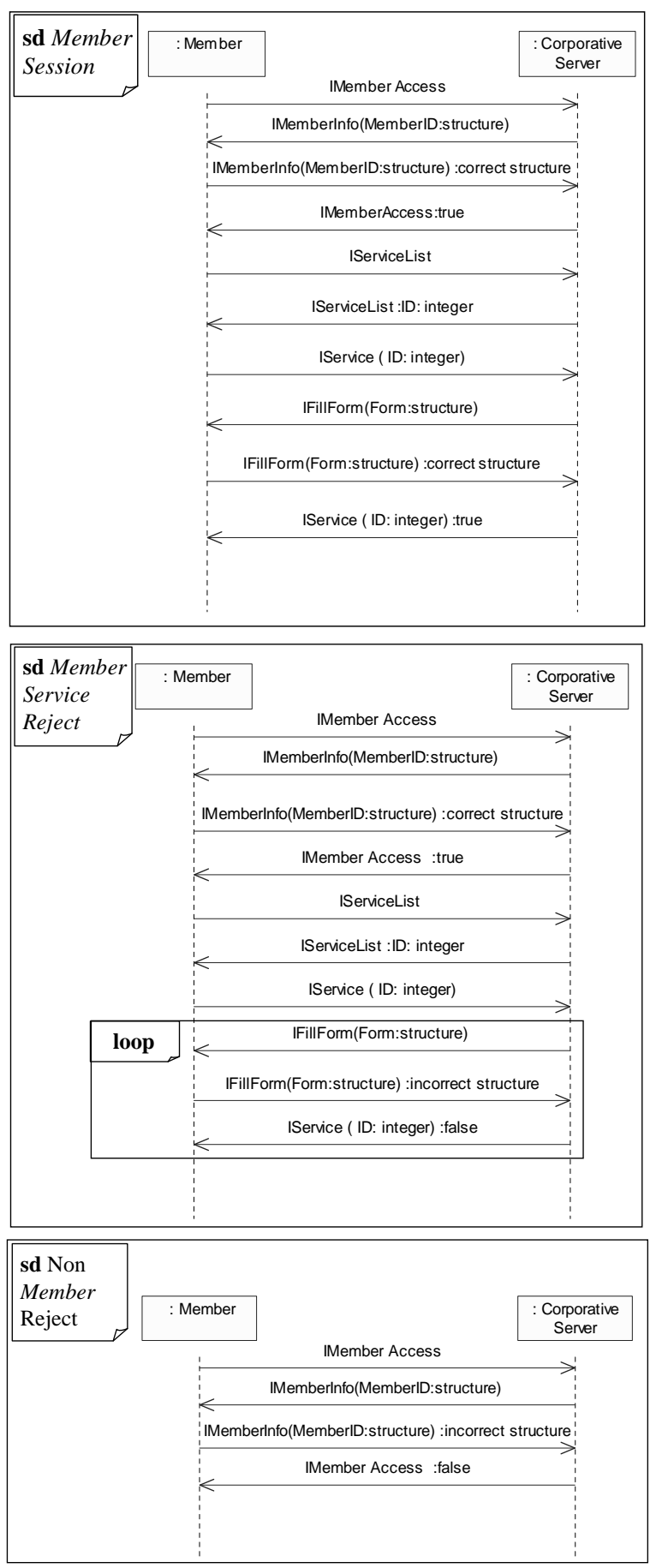

Fig. 3. The sequence diagrams of component Corporative Provider 
- and simplifying the resultant process using axioms of ACP (Algebra of Communicating Processes) with replacement of the parallel composition by the left merge) [9] and axioms for hidden and blocked actions [5]:

$x+\delta=x ; \quad \delta \cdot x=\delta$

$x \cdot \tau=x ; \quad x \cdot(\tau \cdot(y+z)+y)=x \cdot(y+z) ;$

where $x, y, z$ are actions; $\delta$ is a blocked action; $\tau$ is a hidden action.

Intuitively, the blocking of action $a$ means that the process which follows this action will not be considered any more. The hiding of action $a$ makes this action invisible, but the rest of the process which follows this action is taken into consideration.

The above definition is an existential one. The definition leaves unanswered an important practical question: how would we like to inherit the parent process? In other words, the definition given in [5] does not address the tasks of architectural design.

\subsection{Behavioural Inheritance from the Designer's Point of View}

Studying architectural design in practice [15] we have found that designers think about behavioural inheritance in terms of named processes (e.g. "Check Membership" or "Choose a Service from the List"). Composing such processes on the basis of their intuition, designers sometimes make semantic mistakes. So, designers need methodological support to use inheritance intentionally.

In this paper, we propose composing processes in the process tree model. A behavioural inheritance relation on processes of a component-child and a component-parent has to be specified by a constraint in our logic of behavioural inheritance. This logic has the process tree semantics which we define following the semantic definition given in [16].

Let $A P$ be a set of atomic propositions. An atomic proposition $\phi \in A P$ can be of two types: $\beta_{p}=$ " process $p$ begins"; or $\epsilon_{p}=$ "process $p$ ends". So, each atomic proposition has a parameter (the name of the parent process) represented by its index. Each inheritance constraint $\psi$ can be specified by a formula inductively defined as follows:

$$
\psi::=\phi|\neg \psi| \psi_{1} \wedge \psi_{2}\left|\psi_{1} \vee \psi_{2}\right| \psi_{1} A U \psi_{2} \mid \psi_{1} E U \psi_{2} ;
$$

To define the semantics of the logic we construct a Kripke structure [10]: $M=(S P, T, \mu)$, where $S P$ is a finite set of states; $T$ is a binary relation on states which defines the initial state and the possible transitions; $\mu: S P \rightarrow 2^{A P}$ assigns true values of atomic propositions to each state.

The satisfaction relation for formulas in states $(M, s p) \models \psi$ is defined inductively:

1. $s p=\phi$ iff $\phi \in \mu(s p)$.

2. $s p=\neg \psi$ iff $s p \not \models \psi$.

3. $s p=\psi_{1} \wedge \psi_{2}$ iff $s p=\psi_{1}$ and $s p \models \psi_{2}$.

4. $s p=\psi_{1} \vee \psi_{2}$ iff $s p \models \psi_{1}$ or $s p \models \psi_{2}$. 
5. $s p=\psi_{1} A U \psi_{2}$ if for every path $s p_{0}, s p_{1}, \ldots$ with $s p=s p_{0}$, for some $i \geq 0 s p_{i}=\psi_{2}$ and $s p_{j}=\psi_{1}$ for $0 \leq j<i$.

6. $s p_{i} \models \psi_{1} E U \psi_{2}$ if for some path $s p_{0}, s p_{1}, \ldots$ with $s p=s p_{0}$, for some $i \geq 0 s p_{i}=\psi_{2}$ and $s p_{j}=\psi_{1}$ for $0 \leq j<i$.

Using this logic we now give our own definition of behavioural inheritance: behavioural inheritance from the designer's point of view.

Definition 2. Process tree $c$ inherits process tree $p$ if the inheritance constraint $\psi_{p}$ specified for $c$ is satisfied in the root of process tree $c$.

For example, constraint $\beta_{p} A U \epsilon_{p}$ means that process tree $c$ inherits process tree $p$ if for every path of process tree $c$ starting from the root there is a node where process $p$ begins, i.e. $\beta_{p}=$ true, and then there is a node where process $p$ ends, i.e. $\epsilon_{p}=$ true.

The definition of a process $p$ includes a reachability relation on the process's states $s p, s p_{1}, \ldots, s p_{F}$. Let $s p \stackrel{a_{1}}{\Longrightarrow} s p_{1}$ and $s p_{1} \stackrel{a_{2}}{\Longrightarrow} s p_{2}$ specify the reachability relation of a process $p$. This relation is represented by formula $\left(\beta_{a_{1}} A U\left(\epsilon_{a_{1}} \wedge\right.\right.$ $\left.\left.\beta_{a_{2}}\right)\right) A U \epsilon_{a_{2}}$. Recursively applying the formulas of our logic to each reachability relation, it is easy to derive the logic formula which describes the reachability relation of a given process. That is why we use predicate $\Phi_{p}$ to represent a complete process $p$ which starts in state $s p$ and literally fulfils itself without interruptions till its final state. The satisfaction relation for predicate $\Phi_{p}$ is the following:

$s p \models \Phi_{p}$ if for every path $s p_{0}, s p_{1}, \ldots$ with $s p=s p_{0}$, for some $i \geq 0 s p_{i} \models \epsilon_{p}$ and for $0 \leq j<i s p_{j} \models \beta_{p}$ (i.e. $s p \models \beta_{p} A U \epsilon_{p}$ ) and every path $s p_{0}, s p_{1}, . . s p_{i}$ with $s p=s p_{0}$ and $s p_{i}=s p_{F}$ is a path of process $p$.

The logic of behavioural inheritance solves the following problems:

Firstly, this logic allows designers to specify what kind of behavioural inheritance they would like to achieve. Moreover, in the case of composition without modification, the behaviour specified by a constraint can be constructed automatically as a composition of process trees.

Secondly, this logic defines types of constraints ( $A U$ or $E U)$ and allows us to set the correspondence between the type of constraints and the proving technique.

For example, to prove constraint $\beta_{q} A U\left(\epsilon_{q} \wedge \Phi_{p}\right)$ (Fig.4 (1)), we should choose, one after the other, each $s$-tree from process $q$ and block all other s-trees. In the path with the chosen $s$-tree we check that process $p$ starts and literally fulfils itself after finishing of the chosen $s-$ tree. To prove constraint $\beta_{q} E U\left(\epsilon_{q} \wedge \Phi_{p}\right)$ we should check that process $p$ starts and literally fulfils itself for at least one s-tree from $q$ (Fig.4 (2)). For the derivable constraints these two basic techniques are combined.

Sometimes designers need to insert new processes into the parent behavioural patterns. Doing this designers usually mean to keep the parent behavioural pattern in form of one or another inheritance constraint. However, they can make mistakes modifying the parent process. The correctness check of modification (Fig. 5) begins when a starting node of the parent process tree has been found in the child process tree. From this point the tree transformation rules (Fig.6), 


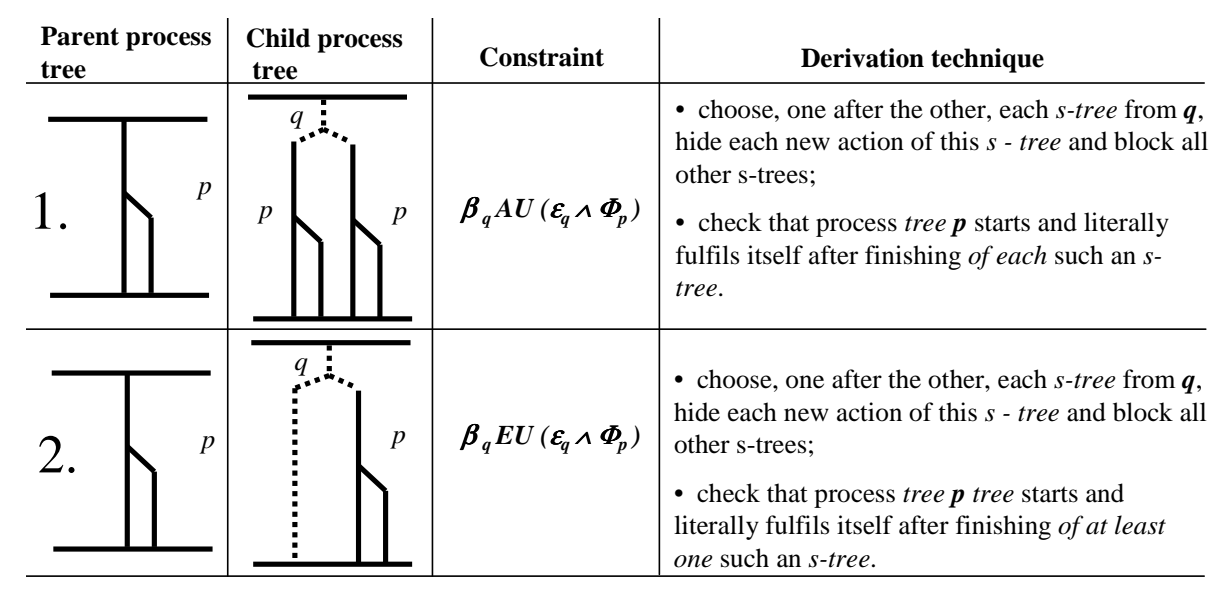

Fig. 4. Examples of Behavioural Inheritance

which we have constructed on the basis of the axioms defined in [5], can be used to check correctness of insertions. Sequential insertions of new actions are hidden (axiom 3). Alternatives started by new actions with the structure corresponding to axiom 4 are transformed according to the transformation rule 4 (Fig.6). Alternatives of other structures starting by new actions are blocked using axiom 1. Axiom 2 is a restrictive one: a blocked action cannot be eliminated from the sequential branch and, this way, the point of incorrect inheritance can be found.

\subsection{An Example of Behavioural Inheritance without Modification of the Parent Process}

The informal inheritance constraint for component Corporative Provider specified in Fig. 2,3 is the following: Only in the case of correct membership, process $p=W e b$ Service is fulfiled without changes. The formal variant of this constraint is: $\left.\epsilon_{s} E U \Phi_{p} \wedge \neg\left(\neg \epsilon_{s} E U \Phi_{p}\right)\right)$.

Component Corporative Provider is a correct inheritor of component Web Service if the process tree of Corporative Provider has a path starting from the root such that there is a node on this path where process $s=$ CorrectMember ship ends and then there is a node on this path where process $p=W e b S e r v i c e$ literally fulfils itself. Also the process tree should not contain paths where there is no end of process $s=$ CorrectMembership but process $p$ fulfils itself.

The process tree representing behaviour of component Corporative Provider is shown in Fig. 7. Thinking in terms of processes designers can "drag-and-drop" processes manually, like in a Lego-game, pointing out the connections between process trees. In such a way the composing of formal constraints can be done automatically by an appropriate tool.

We have developed a prototype of such a tool (see, [17]), which is included into the Rational Rose design environment as an Add-In. The tool allows visualizing 


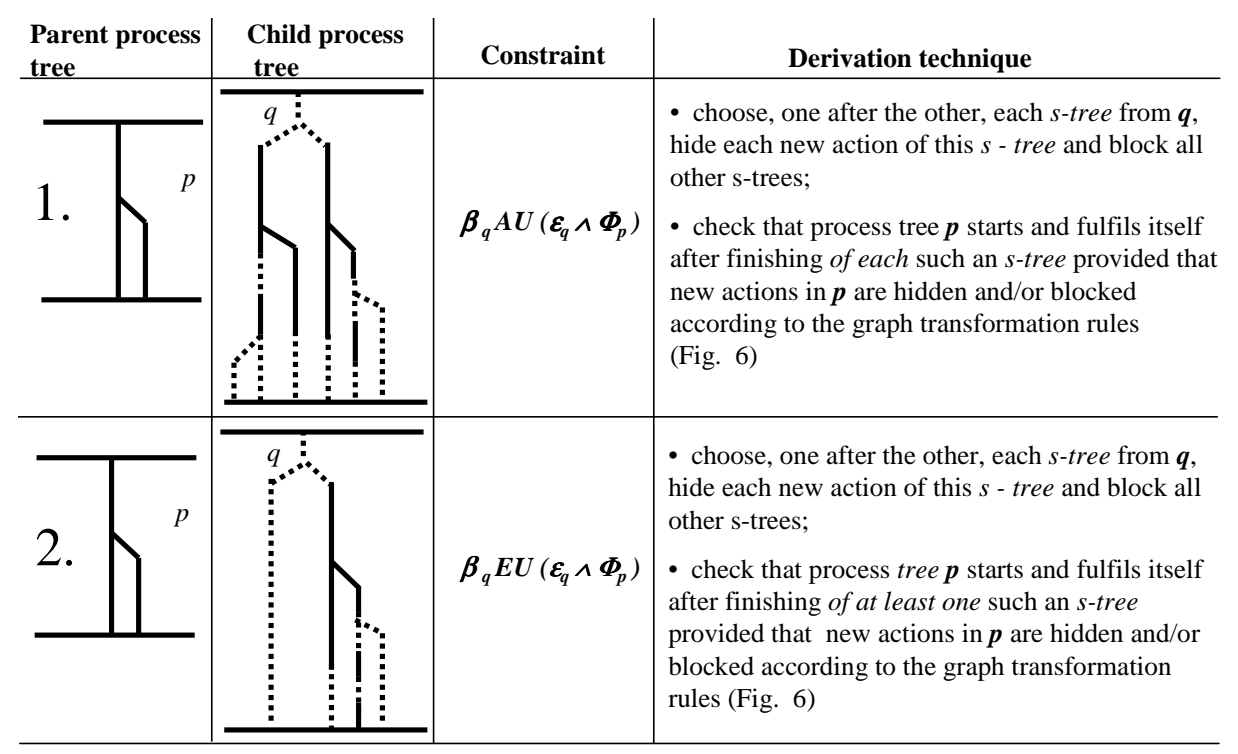

Fig. 5. Examples of Behavioural Inheritance with Modification of a Parent Process

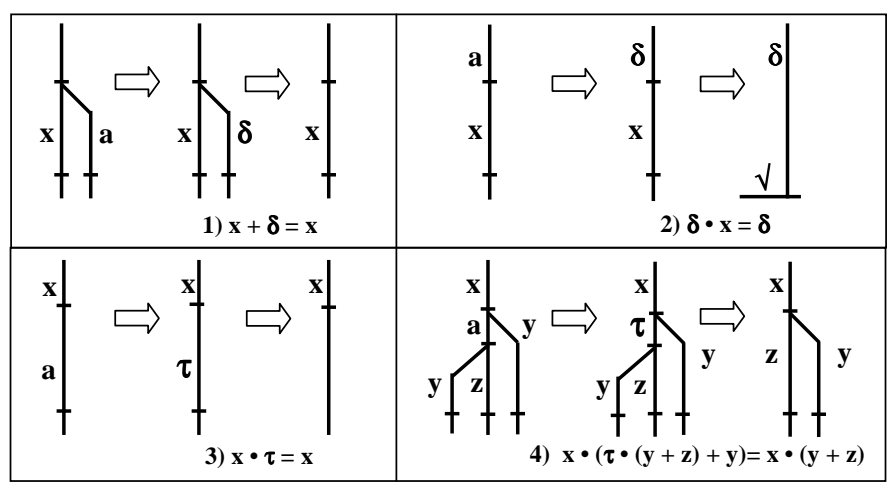

Fig. 6. Graph transformation rules; $x, y, z$ - parent actions; a - new action; $\delta$ - blocked action; $\tau$ - hidden action. 


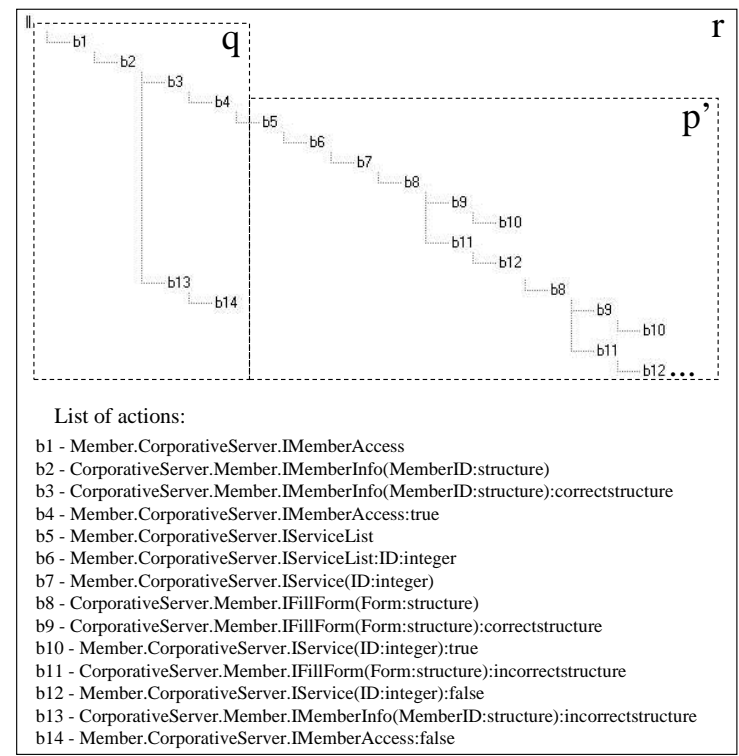

Fig. 7. Process tree of component Corporative Provider. Process $r$ comprises processes $p^{\prime}$ and $q$.

process trees and checking inheritance. The inheritance relation that we have defined on roles in the interface-role diagram (Fig. 2) allows us to duplicate actions of the parent process $p$. Actions of Web Service $a_{1} \ldots a_{4}$ (Fig. 1c) are renamed $b_{5} \ldots b_{8}$ of Corporative Provider (Fig. 7 ); actions $a_{5}, a_{6}$ are renamed $b_{9}, b_{10}$; actions $a_{7}, a_{8}$ are renamed $b_{11}, b_{12}$. Predicate $\epsilon_{q}=$ Correct Membeship ends is satisfied after action $b_{4}$. This path has all states of process $p$ on it. So, according to the given constraint, component Corporative Provider is a correct inheritor of component Web Service.

\subsection{An Example of Behavioural Inheritance with Modification of the Parent Process}

Assume that component Paid Web Service is constructed by inheritance from component Web Service with some altering: after the choice of service, but before getting one a customer should guarantee his payment sending requested information (e.g. a credit card number) back to component Paid Web Service. The new component should be able to utilize process $p=W e b$ Service of component Web Service in the case of confirmed payment represented by some process $c f=$ Confirmed payment. If the payment is not guaranteed, then the session has to be terminated. The informal inheritance constraint may look like this: Only in the case of confirmed payment, component Paid Web Service fulfils process $p=$ WebService. 


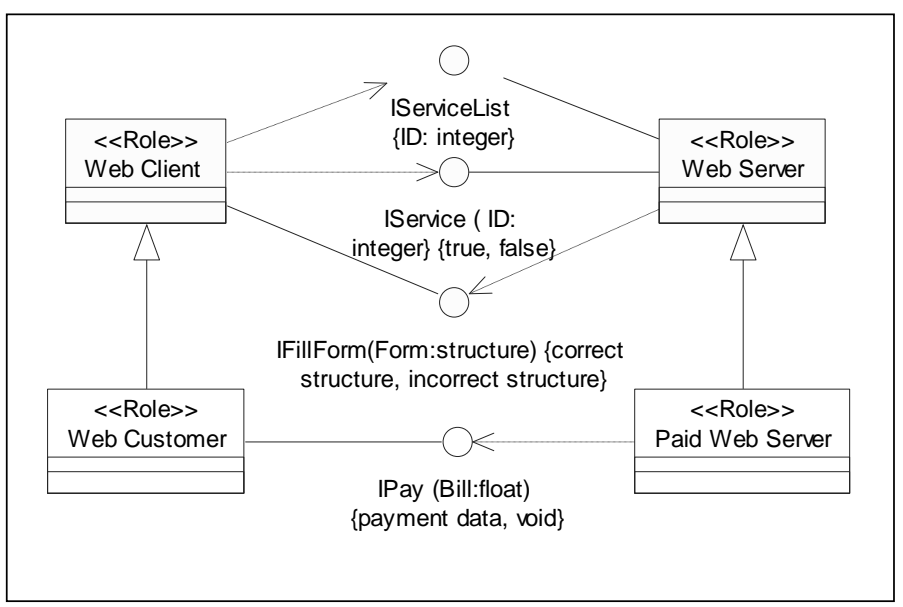

a)
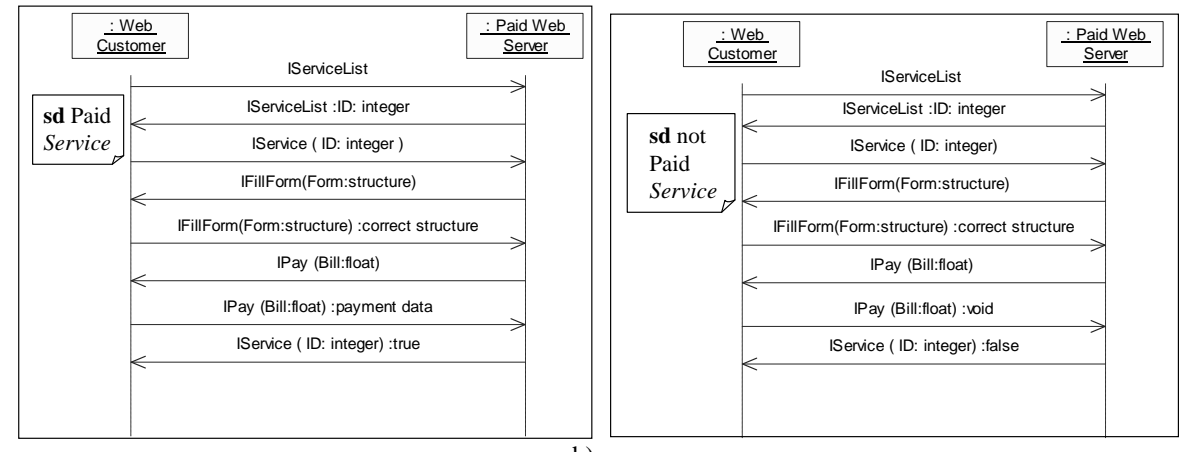

b)

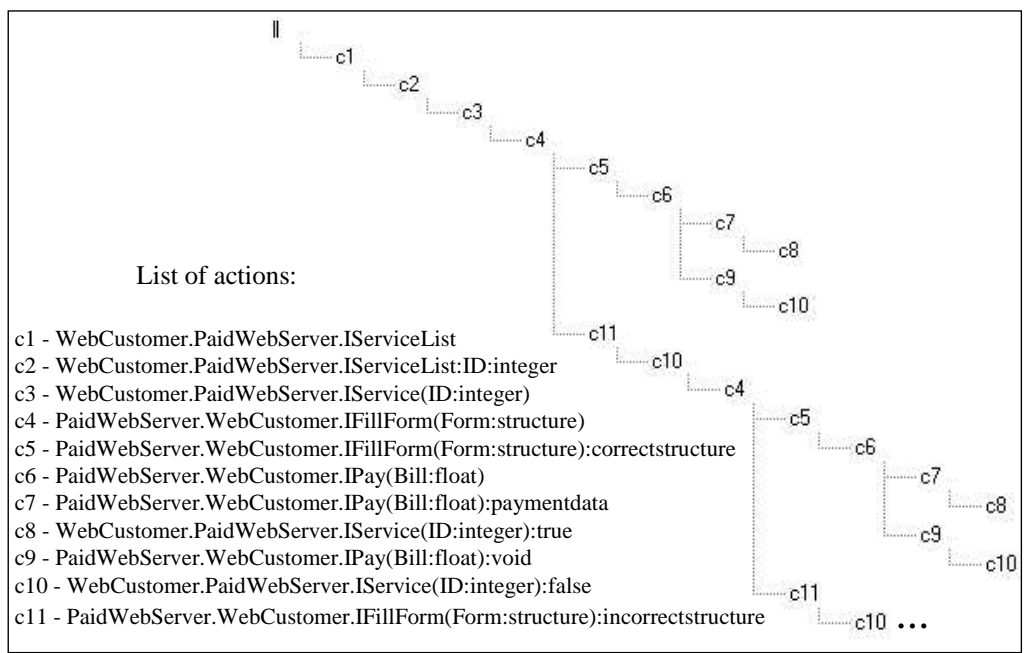

c)

Fig. 8. The specification of component Paid Web Service: a) interface-role diagram; b) sequence diagrams; c) process tree 
The formal variant of the constraint is

$$
\left(\left(\beta_{p} E U \Phi_{c f}\right) A U \epsilon_{p}\right) \wedge \neg\left(\left(\beta_{p} E U\left(\neg \Phi_{c f}\right)\right) A U \epsilon_{p}\right) .
$$

Component Paid Web Service is a correct inheritor of component Web Service if the process tree of Paid Web Service has a path starting from the root such that there is a node on this path where process $p$ begins, then there is a node on this path where process $c f$ begins and literally fulfils itself and then for all paths starting from this node there is a node where process $p$ ends. Also the process tree should not contain paths where process $c f$ is not fulfilled but process $p$ is fulfilled. The specification of component Paid Web Service is shown in Fig. 8.

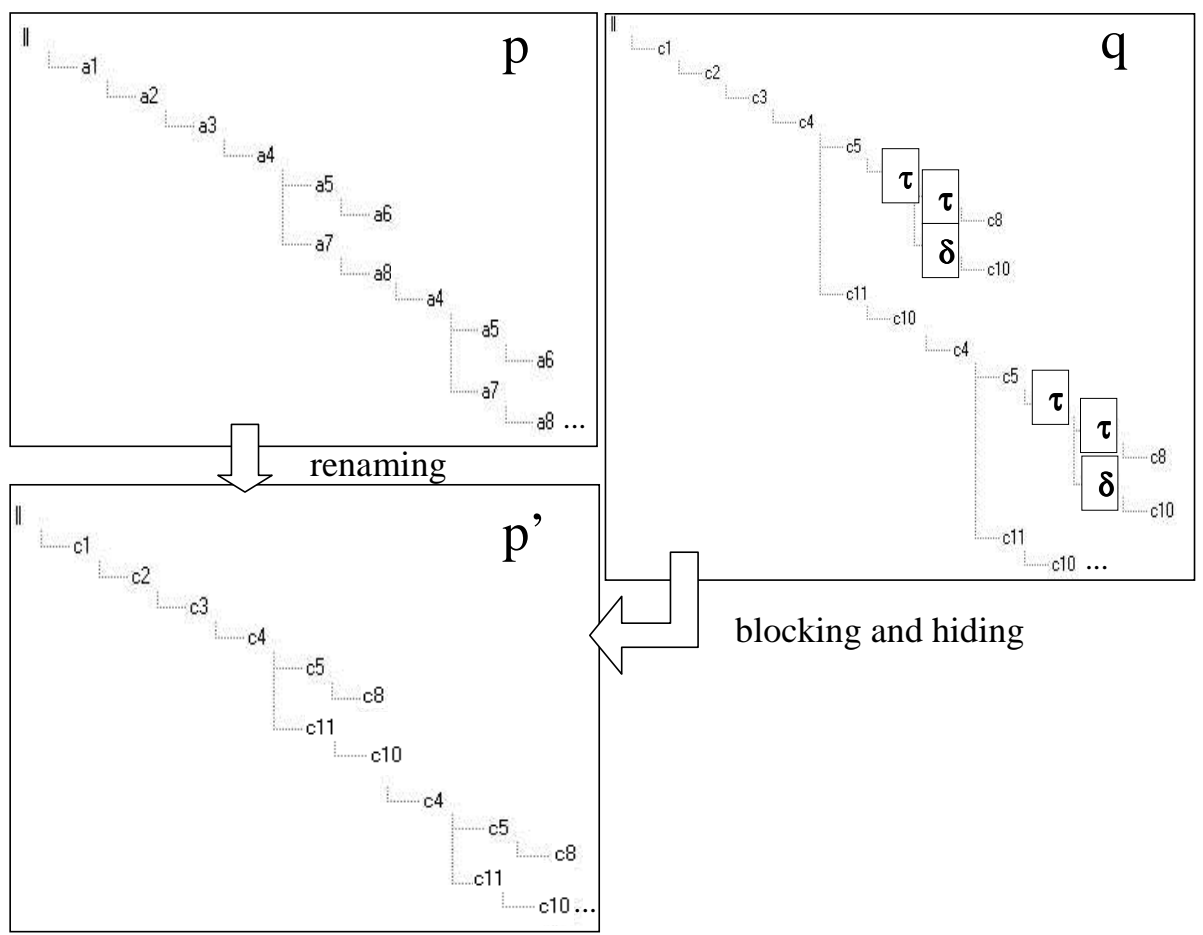

Fig. 9. Paid Web Service inherits Web Service

The interface-role diagram of component Paid Web Service is shown in Fig. 8 a. New roles Web Customer and Paid Web Server inherit corresponding roles of the component-predecessor. One sequence diagram corresponding to the case when the information required to perform the service is incorrect, is completely the same as for the predecessor (see the second sequence diagram in Fig. 1b.) We do not show this case in Fig. 8b. The new behaviour is provided by new interface IPay. Its return value payment data is regarded as a confirmed payment 
and return value void corresponds to a not confirmed payment. Two sequence diagrams (Fig. 8b) show that two actions using interface IPay are inserted between inherited actions. (Compare these two diagrams and the first diagram in Fig. 8).

Process tree $q$ representing behaviour of component Paid Web Service is shown in Fig. 8c. Actions $a_{1}, \ldots, a_{5}, a_{6}, a_{7}, a_{8}$ of component Web Service (Fig. 8) are renamed $c_{1}, \ldots, c_{5}, c_{8}, c_{11}, c_{10}$ (Fig. 9). In the specified process tree of the new component (Fig. 8c) process $p$ starts from the root and we are looking for states where predicates $\beta_{c f}=$ "Confirmed Payment begins " and $\epsilon_{c f}=$ "Confirmed Payment ends" are satisfied. Process $c f=$ Confirmed Payment is a sequence constructed from actions $c_{6}=I$ Pay(bill : float) and $c_{7}=$ IPay(bill : float) : payment data. We choose each such path and continue to investigate it. There exists the final state of process $p$ on each of these paths. So, our inheritance constraint is satisfied. This case is of process modification without composition and the technique with hiding and blocking described in subsection 3.1 can be used to prove the constraint. Our method supports this technique by information to define the actions that should be blocked and the actions that should be hidden. The alternative Not Confirmed Payment is started by action $c_{9}=I$ Pay $($ bill $:$ float $):$ void of new interface IPay. Actions of this alternative are blocked because this process is not considered in our constraint. Actions of process $c f=$ Confirmed Payment are hidden because we consider this process in our constraint. This way, process tree $p^{\prime}=$ WebService' (Fig. 9) is derived. Hence, we may conclude that according to the given constraint component Paid Web Service is a correct inheritor of component Web Service.

\section{Conclusion}

Inheritance of behaviour is a promising technique for component reuse and architectural design. Involving behavioural inheritance in design, we inevitably give birth to additional inheritance constraints on design results and we have to formulate those constraints. This is the price we have to pay to ensure correctness of reuse in design. In this paper, we have proposed to extend existing architectural approaches by specification of how a child-component inherits behaviour of its parent-component. We have defined a logic to represent these relations as behavioural inheritance constraints. The behavioural inheritance constraints can be constructed and proved with the help of tools. The technique described in this paper has been built into the specification tool [17], which we have developed to investigate correctness of components specified by inheritance.

\section{References}

1. Medvidovic N., R.Taylor: A Classification and Comparison Framework for Software Architecture Description Languages. IEEE Transaction on Software Engineering 26 (2000) 
2. D'Souza D.F., A.C.Wills: Objects, Components and Frameworks with UML. The CATALYSIS Approach. Addison-Wesley (1999)

3. Jonkers H.B.M: Interface-Centric Architecture Descriptions. In proceedings of WICSA, The Working IEEE/IFIP Conference on Software Architecture (2001) 113-124

4. Ommering R. van, F. van der Linden, J. Kramer, J.Magee: The Koala component model for consumer electronics software. IEEE Computer 11(3) (2000) 78-85

5. Basten T., W.M.P. van der Aalst: Inheritance of behaviour. The Journal of Logic and Algebraic Programming 46 (2001) 47-145

6. Clarke E.M.,Jr. O. Grumberg, D. A. Peled : Model Checking, Cambridge (1999)

7. Harel D., O. Kupferman: On Object Systems and Behavioural Inheritance. IEEE Transactions On Software Engireering 28 (2002) 889-903

8. OMG: Unified Modeling Language Specification v.1.5, http://www.omg.org/ technology/documents/formal/uml.htm. (2003)

9. Baeten J.C.M.,W.P. Weijland : Process Algebra. Cambridge University Press (1990)

10. Alur R., C.Courcoubetis,D.L.Dill: Model-Checking in Dense Real-Time. Information and Computation 104(1) (1993) 2-34

11. Clark T., A. Evans, S. Kent, S. Brodsky, S. Cock: A Feasibility Study in Rearchitechtoring UML as a Family of Languages using a Precise OO MetaModeling Approach. (2000)

12. OMG: Requirements for UML profiles, OMG document ad99-12-32. (1999)

13. Roubtsova E.E., S.A.Roubtsov: Behavioural Inheritance in the UML to Model Software Product Lines. (Accepted for Elsevier journal "Science of Computer Programming", Editor J. Bosch. (2004))

14. OMG: UML2. http://www.omg.org/uml/. (2003)

15. Roubtsov S.A., E.E. Roubtsova, P. Abrahamsson: Evolutionary product line modelling. In: Proc. International Workshop on Evolution of Large-scale Industrial Software Applications (ELISA), Amsterdam, The Netherlands. (2003) 13-24

16. Manna Z., Pnueli A.: The Temporal Logic of Reactive and Concurrent Systems. V.1. Specification. Springer-Verlag (1992)

17. Roubtsova E.E., S.A.Roubtsov: UML-based Tool for Constructing Component Systems via Component Behaviour Inheritance. ENTCS, Editors T.Erts, W. Fokkink V.80 (2003) 